\title{
Myoepithelial carcinoma inside of maxilla bone: A case report
}

\author{
${\text { FEN } \mathrm{ZHAO}^{1 *}, \mathrm{HONG}^{2} \mathrm{HU}^{2 *} \text { and YING HUANG }}^{1}$ \\ ${ }^{1}$ Department of Pathophysiology, West China School of Preclinical and Forensic Medicine; \\ ${ }^{2}$ Department of Medical Oncology, Cancer Center, West China Hospital, Sichuan University, \\ Chengdu 610041, P.R. China
}

Received August 1,2012; Accepted November 16, 2012

DOI: $10.3892 / \operatorname{mco} .2012 .42$

\begin{abstract}
Myoepithelial carcinoma (MC) is a rare type of carcinoma occurring mainly in the parotid gland, while other carcinomas occur in submandibular or the accessory glands of the oral cavity. They may arise from the glands of the respiratory tract. However, primary $\mathrm{MC}$ of the bone is extremely rare. In the present study, a rare case of a 41-year-old woman with MC in the maxilla bone was reported. Following misdiagnosis as a bone cyst, a local excision was performed. The patient then presented with an extremely high malignancy and showed no response to chemoradiation treatment. The bone cyst was thought to be MC, thus early diagnosis with complete surgical excision was highly important. After confirmation of MC, a secondary extensive surgery may be crucial, following the primary local surgical resection.
\end{abstract}

\section{Introduction}

Myoepithelial carcinoma (MC) is defined as a malignant salivary neoplasm composed almost exclusively of tumor cells with myoepithelial differentiation (1). MC was first described by Stromeyer et al in 1975, and was included in the World Health Organization (WHO) classification of salivary gland neoplasms as a distinct clinicopathological entity, in 1991. $\mathrm{MC}$ is rare, accounting for $\sim 0.4-0.6 \%$ of the salivary gland tumors and $1.2-1.5 \%$ of carcinomas, as noted in a recent large series (2). It arises predominantly from the parotid glands, while other carcinomas occur in the submandibular or the accessory glands of the oral cavity. Such carcinomas may arise from the glands of the respiratory tract (3). However, primary $\mathrm{MC}$ of the bone is extremely rare. In the present study, a case of MC originating from the maxilla bone

Correspondence to: Professor Ying Huang, Department of Pathophysiology, West China School of Preclinical Sciences and Forensic Medicine, Sichuan University, Chengdu 610041, P.R. China E-mail: huangying68@163.com

*Contributed equally

Key words: myoepithelial carcinoma, maxilla, misdiagnosis, malignancy, complete surgical excision showing extremely high malignancy was examined. This study was approved by the Ethics Committee of Sichuan University, Chengdu, China. Informed consent was obtained from the patient.

\section{Case report}

A 41-year-old, previously healthy woman was referred to the Department of Stomatology, West China Hospital (Chengdu, China) due to dysfunction of chewing in the left superior incisor. During clinical examination, a cystic opacity was observed in the left superior section of the maxilla, with a clear smooth boundary and sharp edge. Dental status in other sections of the maxilla was normal. The patient was first diagnosed with a bone cyst of the left maxilla, and received local surgical excision in October, 2007. The post-operative biopsy showed massive muscular spindle cells in combination with small epithelial and plasmacytoid cells. The matrix was mainly myxoid (Fig. 1). The immunohistochemical examination detected the cyst as CK (+); GFAP (+); $\alpha$-SMA $(+++)$; CD10 (-); S-100 (-); and Ki-67, 10\%. The final diagnosis was myoepithelial carcinoma. The patient was then treated with three-dimensional conformal radiotherapy at a dosage of 7000 cGy 30 times, immediately after surgery. However, the patient was suffering from constant, extreme pain in the buttocks 7 months later.

Subsequent to computed tomography (CT) and bone scan examinations showed metastases to the first sacral vertebra, right sacroiliac joint as well as the left anteroinferior iliac spine (Fig. 2). The patient then received radiotherapy for the metastasis at a dosage of $5000 \mathrm{cGy} 25$ times, as well as chemotherapy. The post-treatment CT examination showed progression of the metastasis to the iliac bone. Additionally, certain thoracic and lumber vertebras had also been invaded (Fig. 3A). Several days later, the patient had a pathological bone fracture in the right femoral bone. The patient received chemotherapy a number of times, with several schemes, such as IFO+EPI+DPP, IFO+EPI and paclitaxel+DTIC. However, the progression of cancer could not be controlled. During the upcoming months, the patient presented with paralysis of the right leg, disability of the iliac joints, repeated episodes of high fever, extreme pain, as well as urinary frequency and tenderness. On January 29, 2009, the patient was diagnosed with lung metastasis by CT (Fig. 3B), succumbing to the disease 1 month later. 


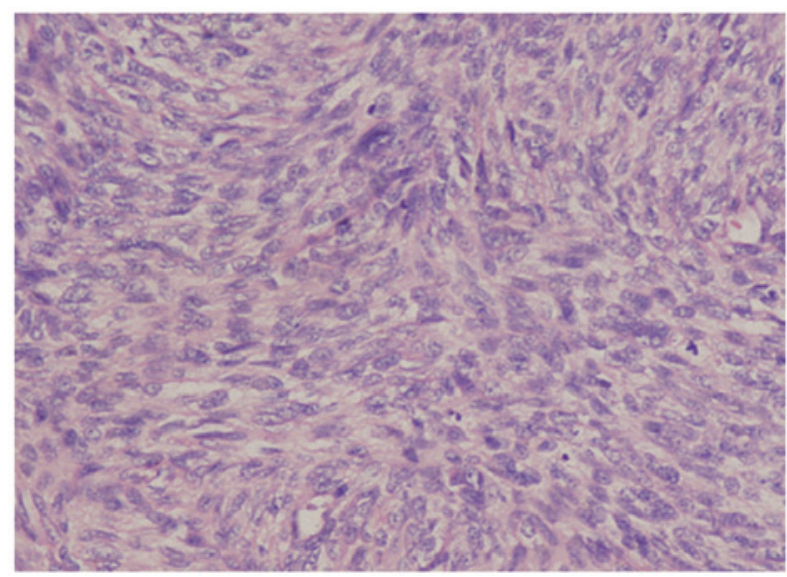

Figure 1. Hematoxylin and eosin (H\&E) staining of pathological slice is shown. The pathological analysis showed that there were massive spindle cells in combination with small epithelial and plasmacytoid cells. The matrix was mainly myxoid (H\&E; magnification, x200).

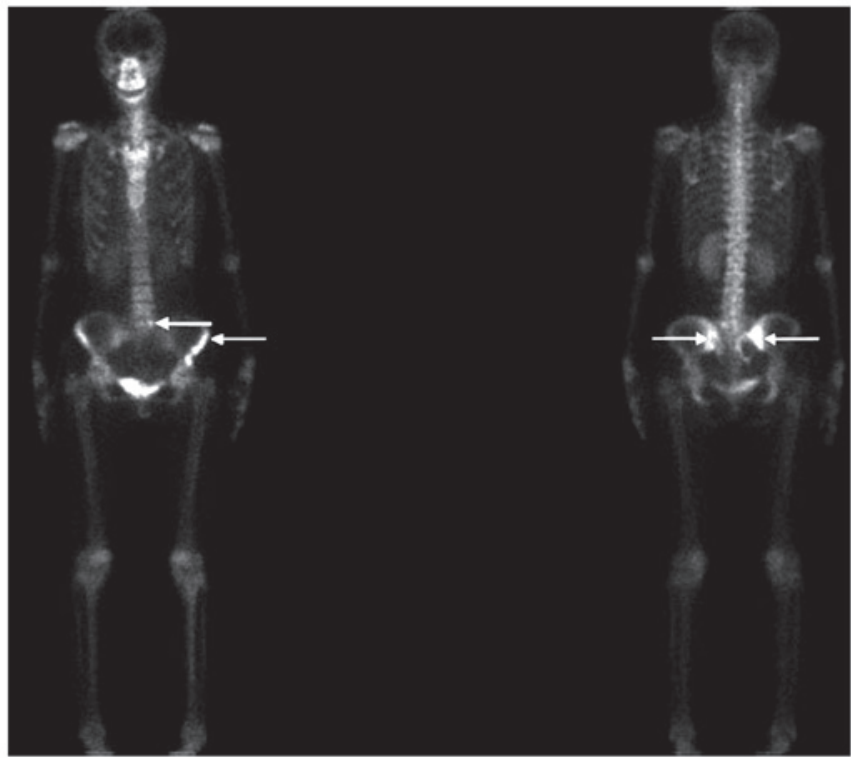

Figure 2. Bone scintigraphy is shown. The bone scan shows abnormally high signal in the first sacral vertebra, right sacroiliac joint and left anteroinferior iliac spine. (arrows).

\section{Discussion}

The diagnosis of $\mathrm{MC}$ is based on two main histologic criteria: unequivocal malignancy and an exclusively myoepithelial nature. The malignancy is supported by the infiltrative growth, the necrotic areas and the cellular pleomorphism in the neoplasm. The myoepithelial nature is suggested by the cytological differentiation of the tumor cells towards myoepithelial elements and the lack of ductal or acinar differentiation (1). The pathological and immunohistochemical examination and clinical process of our patient confirmed the diagnosis of MC.

$\mathrm{MC}$ is an extremely rare type of cancer. It is mainly found in the salivary gland, especially the parotid gland. Although certain cases of the maxillary sinus and hard palate have been
A

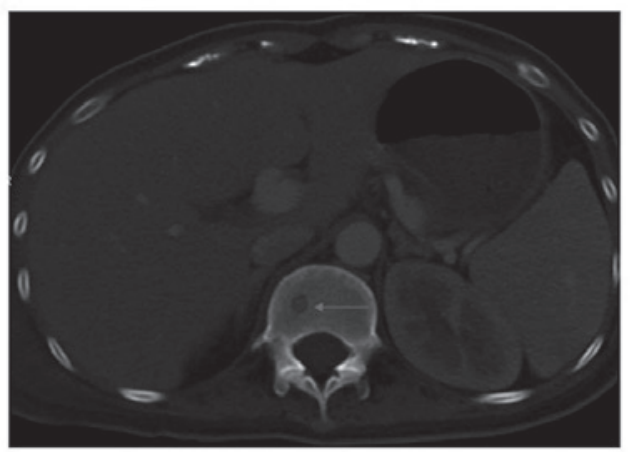

B

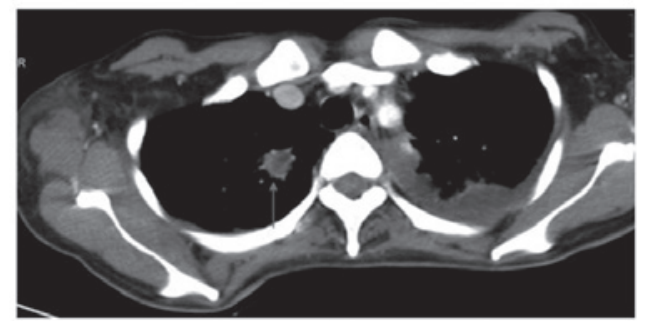

Figure 3. (A) Abdominal and (B) thoracic computed tomography (CT) images are shown. The CT scans detected metastases to the thoracic vertebra and lung (arrows).

reported (3-5), those cases originated from the minor salivary gland on the surface of these bones. The MC of bones in other sites were metastases (6). In the present study, a case of MC located in the left superior part of the maxilla was reported. The MC was extensively treated in our hospital and showed extremely high malignancy and bad prognosis.

The pathogenesis of MC in the bone was unclear, likely because of the presence of residual epithelium in the maxilla bone during the embryonic period. However, in most of these situations, a bone cyst is formed. However, no MC originating from the epithelium within the bone has yet been reported.

The biological behavior and prognosis of MC are not likely to be due to its rarity only. Generally, most MCs showed a low-grade malignancy, although certain reports of MC showed a high-grade malignancy and bad prognosis $(2,7,8)$. As far as the treatment of MC is concerned, experience is limited, with complete surgical excision being mainstay of therapy (8). The role of chemotherapy or radiotherapy in the treatment of MC was unclear $(9,10)$. In this report, the MC of our patient showed an extremely high grade of malignancy. Local surgical excision was performed due to the misdiagnosis as a bone cyst. The patient had distant metastases subsequently, despite the post-operative chemoradiation treatment. Moreover, the disease progressed during the chemoradiation treatment of metastases. These results showed that MC may be extremely malignant. Chemoradiation treatment had limited effect on the treatment of $\mathrm{MC}$, and complete surgical excision was highly important.

In conclusion, in the present study an extremely rare case of MC within the maxilla bone was reported. Following misdiagnosis as a bone cyst, local excision was performed. The patient then presented with an extremely high malig- 
nancy and showed no response to chemoradiation treatment The bone cyst of the oral cavity was likely to be MC, thus early diagnosis with complete surgical excision was extremely important. After confirmation of MC, a secondary extensive surgery may be of great importance, following the primary local surgical resection.

\section{References}

1. Suba Z, Nemeth Z, Gyulai-Gaal S, Ujpal M, Szende B and Szabo G: Malignant myoepithelioma. Clinicopathological and immunohistochemical characteristics. Int J Oral Maxillofac Surg 32: 339-341, 2003

2. Yang S, Li L, Zeng M, Zhu X, Zhang J and Chen X: Myoepithelial carcinoma of intraoral minor salivary glands: a clinicopathological study of 7 cases and review of the literature. Oral Surg Oral Med Oral Pathol Oral Radiol Endod 110: 85-93, 2010.

3. Ren J, Liu Z, Liu X, Li Y, Zhang X, Li Z, Yang Y, Chen Y and Jiang S: Primary myoepithelial carcinoma of palate. World J Surg Oncol 9: 104, 2011.

4. Zhou SH, Ruan LX, Gong L and Wang SQ: Primary malignant myoepithelioma of the left maxillary sinus: a case report. J Int Med Res 36: 362-365, 2008.
5. Hata M, Tokuuye K, Shioyama Y, Nomoto S, Inadome Y, Fukumitsu N, Nakayama H, Sugahara S, Ohara K, Noguchi M and Akine Y: Malignant myoepithelioma in the maxillary sinus: case report and review of the literature. Anticancer Res 29: 497-501, 2009.

6. Bohnstedt BN, Tomcik M, Eads T, Hagen MC and Shah M: Metastasis of soft-tissue myoepithelial carcinoma to clivus. J Neurosurg Pediatr 9: 161-164, 2012.

7. Savera AT, Sloman A, Huvos AG and Klimstra DS: Myoepithelial carcinoma of the salivary glands: a clinicopathologic study of 25 patients. Am J Surg Pathol 24: 761-774, 2000.

8. Kane SV and Bagwan IN: Myoepithelial carcinoma of the salivary glands: a clinicopathologic study of 51 cases in a tertiary cancer center. Arch Otolaryngol Head Neck Surg 136: 702-712, 2010.

9. Kyriazi MA, Carvounis EE, Kitsou M, Arkadopoulos N, Nicolaidou E, Fotiou S and Smyrniotis V: Myoepithelial carcinoma of the vulva mimicking bartholin gland abscess in a pregnant woman: case report and review of literature. Int J Gynecol Pathol 29: 501-504, 2010.

10. Miyata M, Hasegawa K, Ishikawa K, Kato R, Udagawa Y and Kuroda M: Primary myoepithelial carcinoma of the vulva and review of the literature. J Obstet Gynaecol Res 37: 617-622, 2011. 